\title{
Morphological study of cultured preantral ovarian follicles of mice after transplantation under the kidney capsule
}

\author{
Evelyn Telfer*, C. Torrance $\dagger$ and R. G. Gosden \\ Department of Physiology, University Medical School, Teviot Place, Edinburgh EH8 9AG, UK
}

\begin{abstract}
Summary. Isolated ovarian follicles taken from 10-day-old mice and cultured in collagen gel for 5 days, in the presence or absence of serum, were transplanted under the kidney capsule of ovariectomized mice. Hosts showed vaginal opening within 5 days and cornified vaginal smears by 9 days. Follicles proceeded to Graafian stages and luteinization occurred. Ovulation was not observed and oocytes degenerated within the luteinized follicle. Theca formation was preceded by the appearance of blood vessels within the graft. In-vitro fertilisation of harvested oocytes resulted in embryos.
\end{abstract}

Key'words: preantral follicles; collagen gel; culture; in vivo; mouse

\section{Introduction}

The follicle is the functional unit of the mammalian ovary and has a dual role - it provides the micro-environment for oocyte growth and maturation and is responsible for sex steroid production. The follicle develops from the non-growing primordial stage to a large Graafian follicle in a period of approximately 3 weeks according to age (Pedersen, 1970); about two thirds of this phase is spent at the preantral stage. We have described a method for isolating and culturing mouse preantral follicles (Torrance et al., 1989) and demonstrated that ovarian follicles could develop in vitro from small growing stages to large multilaminar follicles. Development was blocked at multilaminar stages and neither theca layers nor antra were formed by these follicles in vitro. These isolated follicles may be abnormal and incapable of becoming Graafian follicles as a result of the conditions in which they were isolated and cultured. Alternatively, the culture system may be inadequate either because of the absence of signals for differentiation or the presence of inhibitors. A method was required to determine whether these follicles were capable of further development. We have now isolated and cultured follicles and transplanted them to the kidney capsule of syngeneic recipients to assess their growth and quality.

\section{Materials and Methods}

Animals. The mice used in this study were $F_{1}$ females aged 8-11 days after birth and derived from matings between $\mathrm{CBA} / \mathrm{Ca}$ males and $\mathrm{C} 57 \mathrm{Bl} / 6$ females. Adult F1 hybrid females were used as hosts for transplants. Hosts were bilaterally ovariectomized at 6 weeks of age and allowed to recover for at least 3 weeks before use. All operations were carried out under intraperitoneal tribromoethanol anaesthesia $(6.03 \mathrm{~g} / \mathrm{kg}$ body $w \mathrm{t})$.

Follicle culture. Follicles were isolated and cultured in a collagen gel matrix as described by Torrance et al. (1989). The animals were killed by decapitation, the ovaries were removed and bisected before being incubated at $37^{\circ} \mathrm{C}$ in $1.5 \mathrm{mg}$ collagenase $/ \mathrm{ml}$ (type 1; Sigma, Poole, Dorset, UK) and $40 \mathrm{DNAse} 1 / \mathrm{ml}$ (Sigma) for $30 \mathrm{~min}$. The follicles were isolated by repeated pipetting of the bisected ovaries using Gilson pipettes. The isolated follicles were pelleted by gentle centrifugation and resuspended in $1 \mathrm{ml}$ Medium 199 (Gibco, Paisley, UK) in a watchglass. Gentle rotation

*Present address: The Jackson Laboratory, Bar Harbor, Maine 04609, USA.

$\Varangle$ Present address: School of Nursing Studies, University of Wales College of Medicine, Heath Park, Cardiff CF4 4XN, UK. 
concentrated the follicles in the centre of the watchglass. Follicles were then transferred to the wells of a Terasaki plate (Flow Laboratories, Irvine, UK) and excess fluid was removed from the wells, $10 \mu$ of the collagen gel solution were added and pipetted once to suspend the follicles. The follicles were incubated for $2-3 \mathrm{~min}$ at $37^{\circ} \mathrm{C}$ until the gel had set. Then $20 \mu \mathrm{l}$ gel solution were pipetted into the empty wells and the $10 \mu \mathrm{l}$ set gels were transferred to them. The double gels were allowed to set as before, then transferred to the wells of a Linbro tissue culture plate (Flow Laboratories) containing $2 \mathrm{ml} \mathrm{M199}$ culture medium supplemented with 5\% donor calf serum (Flow Laboratories). The gels were cultured at $37^{\circ} \mathrm{C}$ with $5 \% \mathrm{CO}_{2}$ in an humidified incubator. The medium was changed at $24 \mathrm{~h}$ and at $72 \mathrm{~h}$.

Morphology of transplanted follicles. A total of 22 gels were obtained from 2 disaggregations using 25 and 26 animals. Follicles were cultured for 5 days and then a single gel was transferred under the kidney capsule of each host animal. Animals were killed between 2 and 21 days after treatment so that an impression of the pattern of growth over this period would be gained. Vaginal smears were taken before and after the collagen gels had been inserted. At the termination of the experiment hosts were killed by cervical dislocation and the kidney was removed. Uteri were removed from all animals and weighed. Each animal was examined to ensure that no ovarian fragments had remained after ovariectomy. The grafts were dissected and fixed overnight in aqueous Bouin's fluid, embedded in paraffin wax and sectioned at $7 \mu \mathrm{m}$. Sections were stained with haematoxylin and eosin and mounted with DPX. Slides were examined for the presence of Graafian follicles and theca layers.

Six ovariectomized animals received control gels and were killed after 12 days; the uteri were removed and weighed. One uterine horn from a control and one from an experimental animal were fixed in aqueous Bouin's fluid and processed for histological examination.

Follicles cultured in a defined medium. The ovaries were removed from seventeen 8-11-day-old F1 mice and follicles isolated as before. Follicles were set in collagen gel and 9 gels were formed; 3 treatment groups containing 3 gels each were established. The control group was cultured in the usual DCS-supplemented Medium 199 and a single gel was harvested at 4,6 and 8 days. A second group was established in the control medium for 4 days and then the gels were washed with serum-free medium and transferred to a well containing Medium M199 supplemented with ITS + (Flow Laboratories). ITS + was used at the recommended level, $1 \mathrm{ml}$ ITS $+/ 100 \mathrm{ml}$ medium, to give a final concentration of $6.25 \mu \mathrm{g}$ insulin $/ \mathrm{ml}, 6.25 \mu \mathrm{g}$ transferrin $/ \mathrm{ml}, 6.25 \mu \mathrm{g}$ selenium $/ \mathrm{ml}, 1.25 \mathrm{mg}$ bovine serum albumin $/ \mathrm{ml}$ and $5.35 \mu \mathrm{g}$ linoleic acid $/ \mathrm{ml}$. Gels from this group were harvested at 6,8 and 10 days. The third group was cultured in Medium M199 supplemented with ITS + from the start of the culture. ITS + and not serum was added to the gel at the polymerization stage for this group. Gels were harvested at 4,6 and 8 days. Gels were fixed and processed for histology as above.

Transplantation of follicles cultured in a defined medium. In a second experiment the ovaries from 19 mice aged 8-11 days were used and follicles isolated as before. Six follicle-dense collagen gels were formed. The culture medium for the gels was supplemented with ITS in place of serum. Cultures of 2 gels per well were set up. Cultures were fed every 2 days. After 5 days in culture the gels were transplanted under the kidney capsule of 6 ovariectomized $F I$ adult hosts. The mice were examined for vaginal opening and vaginal smears were taken daily. One animal was killed 9 days after transplantation and the remaining 5 after 14 days. The grafts were removed and processed for histology. The uteri were also removed and weighed.

Observations on blood vessels and theca. Isolated follicles obtained from 25 animals were placed into 7 gels and after 5 days in culture the gels were transplanted into 7 hosts. One animal was killed on each of the days between 3 and 9 days after transplantation. Grafts were dissected and fixed in formaldehyde for $1 \mathrm{~h}$ followed by formol calcium overnight, embedded in paraffin wax and sectioned at $7 \mu \mathrm{m}$. Alternate ribbons of sections were stained with haematoxylin and eosin or with horseradish peroxidase which stains red blood cells and therefore identifies blood vessels.

In-vitro fertilization of oocytes from grafts. Follicles obtained from 14 animals were placed into 6 gels and cultured for 7 days. Gels were transferred under the kidney capsule of host animals that were ovariectomized at the time of transplantation. Hosts were injected with 10 i.u. PMSG (Organon, Lewes, Surrey, UK) 8 days after transplantation and $48 \mathrm{~h}$ later with 10 i.u. hCG (Organon). Animals were killed $12 \mathrm{~h}$ after hCG injection and the grafts were removed. Fine needles were used to separate the follicles from the gel. Oocytes were aspirated from the largest follicles and fertilized in vitro by established techniques (Wood et al., 1987). Spermatozoa were obtained from a proven fertile Fl hybrid male. Droplets were examined $12 \mathrm{~h}$ later and the number of 2-cell embryos was counted.

\section{Results}

\section{Morphology of transplanted follicles}

After ovariectomy normal vaginal smear patterns were abolished and the vagina closed. The vagina began to open within 3 days of transplanting follicles under the kidney capsule and the vaginal epithelium was cornified after 7 days. Rather than presenting short regular cycles of cornification the animals exhibited persistent cornification. No vaginal opening was observed in control 


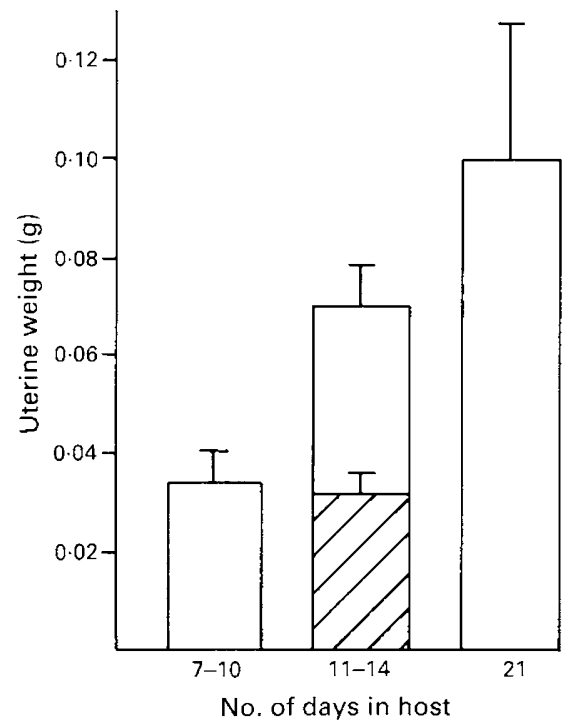

Fig. 1. Histogram showing mean \pm s.e.m. uterine weight of ovariectomized animals after transplantation of cultured follicles (open bar) or collagen gel controls (hatched bar).
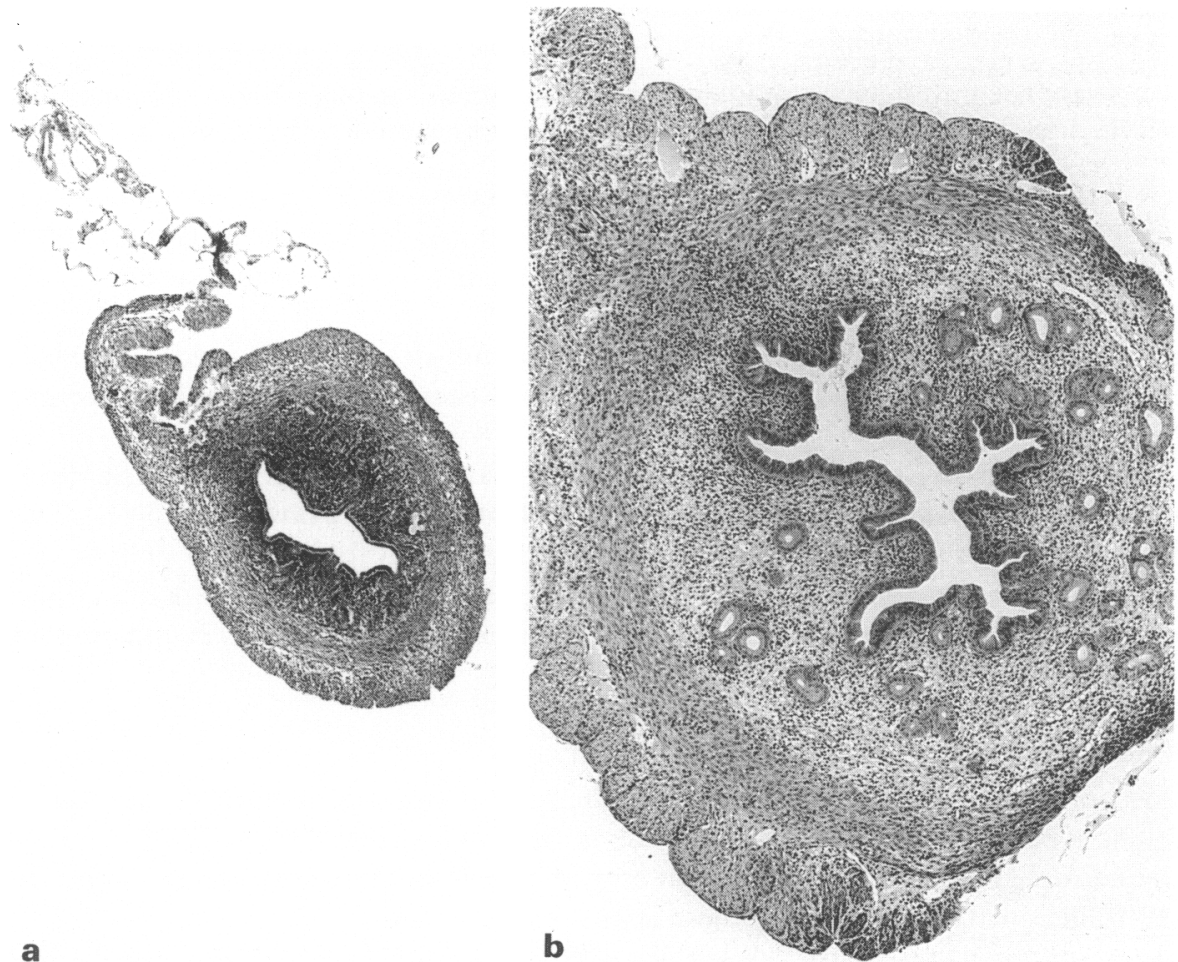

Fig. 2. Photomicrograph of uterine cross-sections from ovariectomized animals (a) 21 days after gel-only (control) transplantation and (b) 21 days after transplantation of cultured follicles under the kidney capsule. 

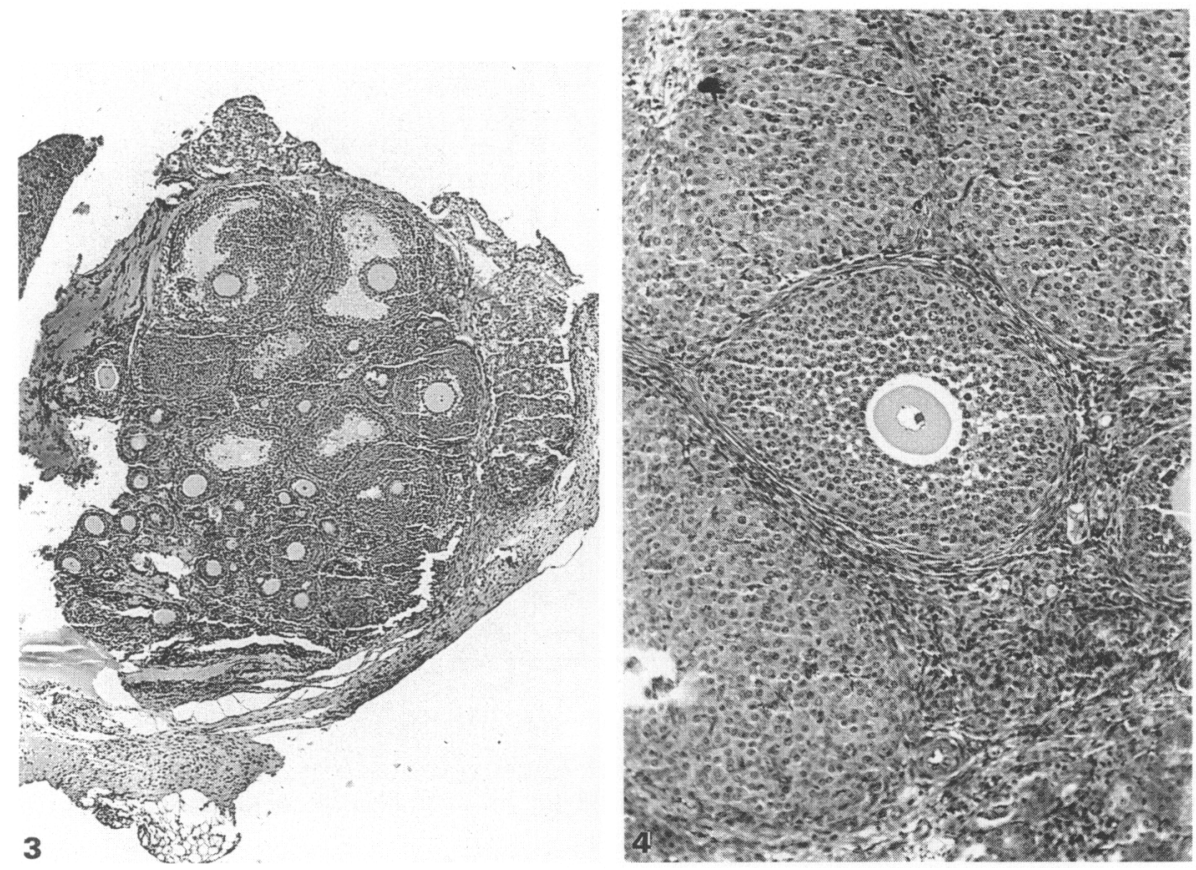

Fig. 3. Section of a collagen gel containing follicles grafted under the kidney capsule $(\times 50)$. Follicles were cultured for 5 days before transplantation and the graft removed after 13 days in the host. All stages of follicular development can be seen.

Fig. 4. Section of graft showing multilaminar follicle with well defined theca layer $(\times 160)$. This follicie had been cultured for 5 days and under the kidney capsule for 13 days.

animals. Uterine weights showed a significant increase compared with control animals between 11 and 14 days after transplantation (Fig. 1). The control uterus showed the expected hypotrophy of the ovariectomized state (Fig. 2a) whilst the experimental uterus showed the uterotrophic effects of oestrogen (Fig. 2b).

After the culture period and before transplantation, follicles in the gels had developed to large preantral stages but no antra or thecal development had occurred (as reported by Torrance $e t$ al., 1989).

The grafts were easily identified under the kidney capsule. All stages of follicle development from primordial to Graafian were identified in the grafts from Day 7 onwards (Fig. 3). Thecal cells were observed in multilaminar follicles (Fig. 4) and large antral follicles were present (Figs 5, 6). Despite the presence of mature follicles the grafts were always anovulatory. Graafian follicles were undergoing atresia with degeneration of the oocytes within the follicles (Fig. 7) and luteinization of granulosa cells (Fig. 8).

\section{Follicles cultured in defined medium}

Microscopic assessment of the follicles from the control and experimental groups did not reveal any differences between the morphology of the follicles cultured in ITS or DCS. After 5 days in serum-free culture growth had proceeded but only to large multilaminar preantral stages. No antral follicles or follicles with a well defined theca layer were observed.

\section{Morphology of follicles after transplantation}

In the group with follicles transplanted after culture in a defined medium, vaginal opening occurred in all animals after 6 days. Vaginal smears showed evidence of cornification and uterine 

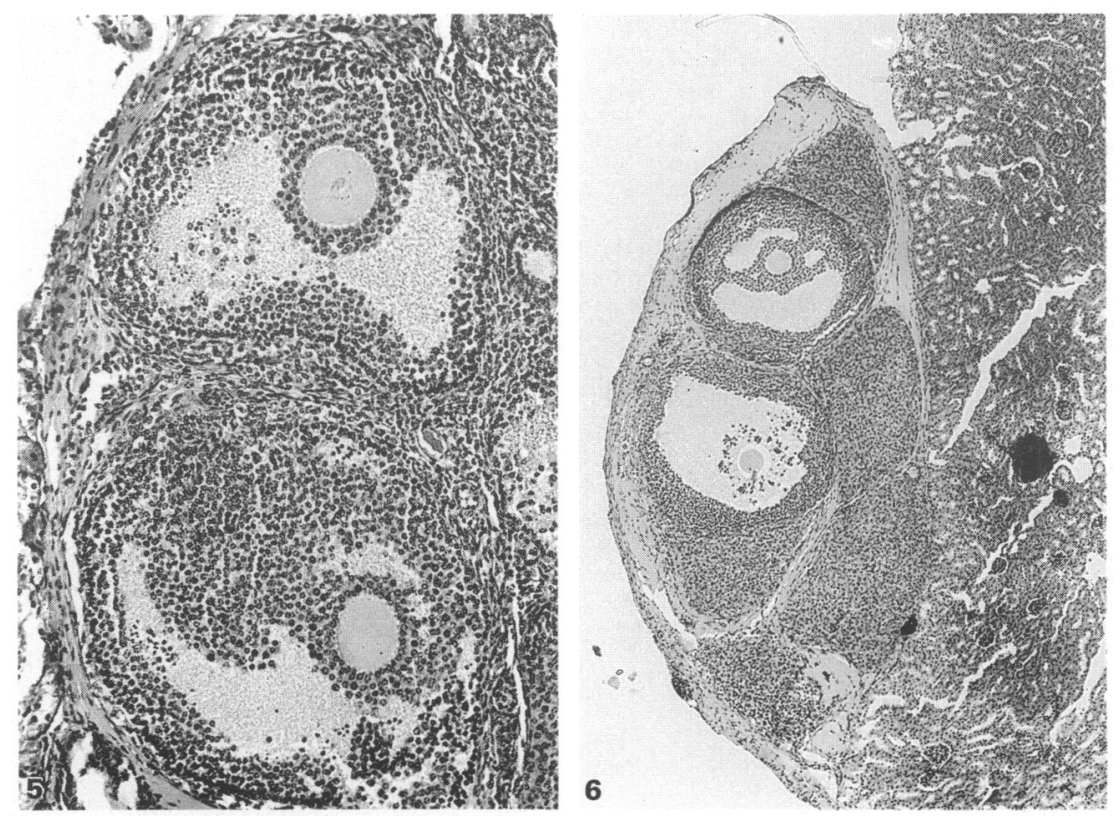

Fig. 5. Section of graft showing large Graafian follicles after 5 days in culture and 13 days under the kidney capsule $\times 160$.

Fig. 6. Section showing large antral follicle and degenerating Graafian follicle $(\times 50)$. Follicles had been cultured for 5 days before transplantion and kept under the kidney capsule for 21 days.

weights increased significantly. Microscopy demonstrated the presence of a range of growing follicles including large antral follicles. These grafts were morphologically similar to those from follicles cultured in the presence of serum.

\section{Observations on blood vessels and theca}

After 3 days in vivo there was no evidence of theca cells or blood vessels in the graft. Some small blood vessels were apparent by 5 days but theca layers were not present around follicles. On Day 6 both theca and blood vessels were present and by 7 days both were well represented.

\section{In-vitro fertilization of oocytes from grafts}

Altogether 16 oocytes were recovered from 6 gels and fertilized and 12 developed to the 2-cell stage whilst 4 formed blastocysts.

\section{Discussion}

In this study we have shown that small preantral follicles from immature animals grown in vitro with or without serum are capable of progressing to the mature Graafian stage when transferred under the kidney capsule. Within 5 days these transplanted follicles were producing sufficient oestrogen to support vaginal opening in ovariectomized animals and after 7 days cornification of the vaginal epithelium was established. These results would indicate that the follicles are capable of developing beyond the multilaminar stages and that the limiting factor to further development in vitro appears to be the culture conditions. 

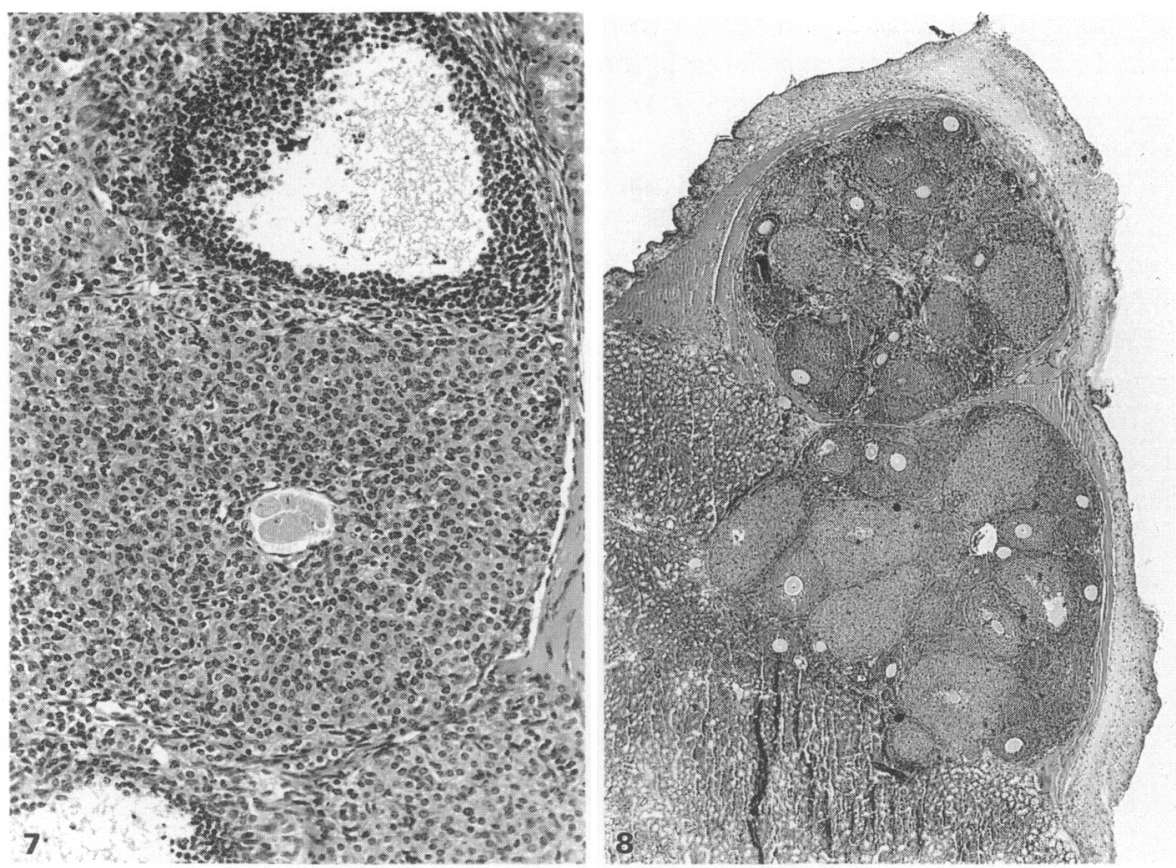

Fig. 7. Degenerating follicle with trapped oocyte $(\times 160)$. Follicles were cultured for 5 days before being transferred to the kidney capsule. This graft was removed after 13 days in the host.

Fig. 8. Section of a graft showing luteal like structures $(\times 35)$. Follicles were cultured for 5 days before transplantation and removed from the host after 15 days.

The results from using a partly defined medium show that follicles are capable of growing to large preantral stages without serum. The follicles appear by light microscopy to develop in a similar way to follicles in serum-supplemented media. When the follicles grown in defined medium were transplanted under the kidney capsule, they developed to large antral stages, indicating retention of their developmental potential. Follicle growth in a defined medium therefore represents a potentially powerful tool for investigating hormonal and growth factor effects on follicle development in vitro.

All stages of follicular development were present in the grafts. Even after 21 days in vivo primordial and small growing follicles of 1 and 2 granulosa cell layers were present. This suggests that the growing follicles had recently been recruited from the primordial pool. Collagenase dissociation and 5 days in vitro had not restricted the growth potential of the primordial follicles. The grafts appeared to remain as discrete units under the kidney capsule with no evidence of invasion by kidney cells and the grafts could be easily separated from the kidney tissue. This suggests that the differentiation of the theca and the appearance of antra were due to the endocrine environment in vivo and not to local interaction with the kidney cells.

In vivo thecal differentiation appears as the follicle reaches the 4 granulosa cell layer stage with antrum formation occurring at Day 13-15 (Gosden et al., 1988). The presence of the theca would appear to be necessary for further follicle growth and the steroidogenic capability of the follicle is expected to be compromised when theca layers are absent (Richards \& Kersey, 1979). It has been suggested that thecal cell differentiation is related to subtle increases in serum LH concentrations (Bogovich \& Richards, 1982). While thecal cells were absent at the beginning of the culture, stromal cells, which are assumed to be their precursors, were present. The conditions in vitro were not optimum for the differentiation of stroma to theca. The results of our study show theca developing 
only after the appearance of blood vessels in the graft. Endothelial cell contact or blood borne factors may be important in theca differentiation.

Ovulation did not occur from the grafts, oocytes degenerated within the follicle and granulosa cells luteinized. This pattern of follicular degeneration has been observed in the rat ovary (Popkin et al., 1983) and in the mouse after suppression of ovulation by progesterone (Telfer, 1987). Intact ovarian fragments transplanted under the kidney capsule ovulate (Felicio et al., 1983) and it could be that the lack of a surface epithelium in the grafts explains these differences.

Embryos were produced from oocytes taken from follicles grown in vitro and matured in vivo, showing that the culture of immature follicles does not impair their developmental potential. Using the same culture and transplantation method with frozen preantral follicles Carroll et al. (1989) have produced live young. Live mice have also been produced from follicles cultured under different conditions (Eppig \& Schroeder, 1989).

Studies with cultures of preantral hamster follicles have shown that antral development does not occur in vitro unless FSH is present in the medium (Roy \& Greenwald, 1989). We have conducted preliminary experiments with FSH, LH and EGF but as yet we have not observed any antral development in vitro. The techniques of follicle isolation and culture described in our previous paper (Torrance et al., 1989) and the assessment of cultured follicles after transplantation in vivo provide the opportunity to study the factors influencing the development of follicles from the primordial to preantral stage. With further development of the techniques it may be possible to produce all stages of follicular development in vitro.

We thank Kay Grant for technical assistance with the histology; the Faculty of Medicine, University of Edinburgh, for a scholarship to C.T.; and the Wellcome Trust for a grant to R.G.G.

\section{References}

Bogovich, K. \& Richards, J.S. (1982) Effects of human chorionic gonadotropin and progesterone on follicular development in the immature rat. Endocrinology 111, 1429-1438.

Carroll, J., Wood, M., Telfer, E., Gosden, R.G. \& Whittingham, D.G. (1989) Freeze preservation and subsequent development of immature follicles. Proc. Eur. Soc. Hum. Rep. Endocr. Abstr.

Eppig, J.J. \& Schroeder, A.C. (1989) Capacity of mouse oocytes from preantral follicles to undergo embryogenesis and development to live young after growth, maturation and fertilization in vitro. Biol. Reprod. 41, 268-276.

Felicio, L.S., Nelson, J.F., Gosden, R.G. \& Finch, C.E. (1983) Restoration of ovulatory cycles by young ovarian grafts in aging mice: potentiation by longterm ovariectomy decreases with age. Proc. natn Acad. Sci. USA 80, 6076-6080.

Gosden, R.G., Hunter, R.H.F., Telfer, E., Torrance, C. \& Brown, N. (1988) Physiological factors underlying the formation of ovarian follicular fluid. J. Reprod. Fert. 82, 813-825.

Pedersen, T. (1970) Determination of follicle growth rate in the ovary of the immature mouse. J. Reprod. Fert. 21, 81-93.

Popkin, R.M., Fraser, H.M. \& Gosden, R.G. (1983) Effects of LH-RH agonist on LH-RH immunoneutral- ization on pituitary and ovarian LH-RH receptors in female rats. J. Reprod. Fert. 69, 245-252.

Richards, J.S. \& Kersey, K.A. (1979) Changes in theca and granulosa cell function in antral follicles developing during pregnancy in the rat: gonadotropin receptors, cyclic AMP and estradiol 17ß. Biol. Reprod. 21, 1185-1201.

Roy, S.K. \& Greenwald, G.S. (1989) Hormonal requirements for the growth and differentiation of hamster preantral follicles in long-term culture. J. Reprod. Fert. 87, 103-114.

Telfer, E. (1987) Factors influencing follicular development in mammalian ovaries. Ph.D. thesis, University of Edinburgh.

Torrance, C., Telfer, E. \& Gosden, R.G. (1989) Quantitative study of the development of isolated mouse pre-antral follicles in collagen gel culture. J. Reprod. Fert. 87, 367-374.

Wood, M.J., Whittingham, D.G. \& Rall, W.F. (1987) The low temperature preservation of mouse oocytes and embryos. In Mammalian Development: A Practical Approach, ch. 13, pp. 255-279. Ed. M. Monk. IRL Press, Oxford. 